\title{
2015 Mathematics Programs That Make a Difference
}

Each year the AMS Committee on the Profession (CoProf) selects outstanding programs to be designated as Mathematics Programs That Make a Difference. For 2015 CoProf selected two programs: the CENTER FOR UNDERGRADUATE RESEARCH IN MATHEMATICS (CURM) AT BRIGHAM YOUNG UNIVERSITY and the PACIFIC COAST UNDERGRADUATE MATHEMATICS CONFERENCE (PCUMC).

\section{Citation: CURM}

Be it resolved that the American Mathematical Society and its Committee on the Profession recognize the Center for Undergraduate Research in Mathematics (CURM), housed in the Department of Mathematics at Brigham Young University, for its significant efforts to encourage students from underrepresented groups to continue in the study of mathematics.

CURM provides training and funding for mathematics faculty at institutions across the United States to engage small groups of undergraduates in research during the academic year, with an emphasis on recruitment from underrepresented groups and historically minority-serving institutions. Since its creation in 2007, 312 students and 96 faculty members from 89 different institutions have participated in the program. Faculty begin their engagement with CURM by attending a three-day summer workshop where they receive extensive advice on mentoring undergraduate research. After returning home, CURM faculty conduct research projects for groups of two to five students, culminating in an annual conference where the students present their findings. These efforts have yielded 263 conference presentations and 32 published research papers by CURM students. Nearly two-thirds of CURM alumni have gone on to graduate school, with many alumni attesting that their experience with CURM played a major role in that decision, while CURM faculty report that the program has a significant and lasting impact on the practices and cultures of their departments. Several institutions have replicated the CURM model, on a smaller scale, to develop mentoring

DOI: http://dx.doi.org/10.1090/noti1253 programs and research opportunities for all their mathematics students. CURM has been supported by the Division of Mathematical Sciences of the National Science Foundation.

The AMS commends the Center for Undergraduate Research in Mathematics and its administrators for their high level of commitment and successful efforts to improve diversity in the profession of mathematics in the United States.

\section{About CURM}

In the last couple of decades, Research Experiences for Undergraduates (REU) programs have multiplied. Often with funding from the National Science Foundation (NSF), REU programs typically bring undergraduate mathematics majors to a college or university campus for an intensive research experience lasting several weeks over the summer. The REU model has been highly successful, creating a lasting impact on students, many of whom continue to doctoral programs in the mathematical sciences or other areas, as well as on to the faculty of the institutions where the REU programs are held.

The Center for Undergraduate Research in Mathematics (CURM), founded in 2007 in the mathematics department at Brigham Young University, operates on a model complementary to that of traditional REUs. CURM provides support and training to faculty so that they can build, at their home institutions, small groups of two to five undergraduate students to work on research projects during the academic year. With a focus on minority-serving institutions, CURM has two main aims. The first is to get undergraduate students excited about research and to encourage them to finish their degrees and consider graduate school. The second is to help untenured faculty successfully negotiate the critical career transition point at which they become full-fledged mathematics professors.

Faculty apply to participate in CURM, and those who are accepted receive "mini-grants" that range from US\$15,000 to US\$25,000. The mini-grants provide stipends for participating faculty and 
students, travel, and supplies. CURM is funded by two grants totaling US\$2.6 million from the NSF's Division of Mathematical Sciences, as well as support from the VWR Foundation and Brigham Young University. Participating faculty come to Brigham Young for a three-day workshop designed to train them as mentors to undergraduate students doing research. After the faculty return to their home institutions, CURM provides them with support and advice throughout the academic year as they work on research projects with their students. Faculty return to Brigham Young, together with their student groups, for CURM's annual Student Research Conference, where students present their findings.

"I cannot think of a mathematics program that has made a larger difference in undergraduate mathematics research than [CURM]," wrote Kathryn Leonard in a letter supporting CURM for the Programs That Make a Difference award. Leonard, who is at California State University-Channel Islands, received CURM grants for two years. "CURM transformed the lives of my students, all of whom were from underrepresented groups or were first-generation college students.... Their experience with CURM built confidence, experience and a sense of their place in the mathematical world."

CURM has had a profound and widespread effect. Since its inception, the center has provided financial support to over three hundred undergraduate students (48 percent female, 26 percent minority, and 21 percent first-generation college students). CURM students have written 130 joint research papers and have given over 250 single or joint conference presentations or poster presentations. Their work has garnered a total of thirty awards. What is more, CURM gives undergraduates the inspiration and the confidence to consider graduate education. Among all mathematics majors at CURM-participating institutions, only 18 percent go on to graduate school. By contrast, 63 percent of CURM students do so.

The effects of CURM on faculty have also been significant. Almost one hundred faculty members from close to ninety institutions have participated in CURM. After completing the CURM year of mentoring, over 90 percent of them have continued to work with undergraduates in research. These faculty members have also been energized to change the practices and cultures within their own departments to emphasize excellent teaching and mentoring and to encourage students to reach their highest potential.

"[T]he CURM program has been highly effective in bringing the full undergraduate research experience to students and faculty at institutions [like mine]," wrote Rebecca Garcia of Sam Houston State University in a letter supporting CURM's nomination for the award. Using her CURM experience and with funding from the NSF and from the National Security Agency, Garcia launched PURE
Math, (Pacific Undergraduate Research Experience in Mathematics), the first and only mathematics undergraduate research program located in the US Pacific Islands. Garcia, who was born and raised in Guam, is probably the first female Pacific Islander to receive her doctoral degree in pure mathematics and the only female Pacific Islander mathematician in higher education.

Leonard also successfully applied for funding from the NSF to launch an REU at her home institution. "The simplicity and effectiveness of the CURM model to inspire and sustain active undergraduate research programs is unmistakable, and is particularly noteworthy at schools where research might not be a high priority," Leonard said in her letter. "Most important, CURM provides a life-changing experience for students who need it most. CURM truly makes a difference."

\section{CURM Director: Michael Dorff, Brigham Young University}

\section{Citation: PCUMC}

Be it resolved that the American Mathematical Society and its Committee on the Profession recognize the Pacific Coast Undergraduate Mathematics Conference (PCUMC) for its significant efforts to encourage students from underrepresented groups to continue in the study of mathematics.

PCUMC is an annual one-day student conference held at a rotating group of colleges and universities in the greater Los Angeles area. The conference gives undergraduates the opportunity to share research and expository projects, and aims to encourage the participation of underrepresented minorities and women in the undergraduate mathematical community. Since its founding in 2006, the PCUMC has hosted 2,400 participants (including nearly 400 student speakers) from more than one hundred different institutions, of which thirty-seven are Hispanic-Serving Institution members of the Hispanic Association of Colleges and Universities. In addition to student presentations and carefully selected keynote speakers, the conference features panel discussions on topics such as graduate programs, summer internship and research opportunities, and career options in the mathematical sciences. Many of the keynote speakers and panelists are from industry. The student participants praise the conference's welcoming and supportive environment, and faculty at participating institutions laud PCUMC as one of the most significant events for their mathematics majors each academic year. PCUMC has had a substantial impact on undergraduate mathematics in southern California, and provides a model that could be adopted in other metropolitan areas.

The AMS commends the Pacific Coast Undergraduate Mathematics Conference and its organizers for their high level of commitment 
and successful efforts to improve diversity in the profession of mathematics in the United States.

\section{About PCUMC}

On March 14, 2015, over six hundred students and faculty from colleges and universities across southern California and Nevada descended on the campus of California Lutheran University in Thousand Oaks for the tenth annual Pacific Coast Undergraduate Mathematics Conference (PCUMC). This particular installment of the annual PCUMC was special for at least two reasons. First, it was held on "Super Pi Day," the date of which can be written as 3.14.15, representing the first five digits of $\pi$. Second, in recognition of the one-hundredth anniversary of the Mathematical Association of America (MAA), the conference was held jointly with the MAA's Southern California-Nevada Section. In addition to screenings of the MAA Centennial video, the PCUMC featured $\pi$-themed plenary lectures, student poster and talk sessions, and panel discussions, as well as more light-hearted events like the "pi-athon" session in which participants recited the digits of $\pi$ for the sheer fun of it.

Since its establishment in 2006, the PCUMC has become an annual event eagerly anticipated by students and faculty in the greater Los Angeles area. The number of participants has grown from 86 in the first year to a high of 650 in 2012. The goal of the PCUMC is to provide support and mentoring to undergraduate students who are the future members of the mathematical community. The conference brings the students into contact with outstanding role models and raises awareness about career options and advanced study in the mathematical sciences. In the friendly, supportive atmosphere of the PCUMC, students can make presentations about expository mathematics or about their own work, get to know others who share their interest in mathematics, hear high-quality lectures by senior mathematicians, and attend panel discussions about graduate school and careers. In addition, the PCUMC makes a special attempt to reach out to younger students by including special freshman and sophomore talk sessions.

The granddaddy of all undergraduate mathematics conferences is no doubt the Hudson River Undergraduate Math Conference, which started in 1994 and nowadays draws around four hundred participants each year. In 2003, with a grant from the National Science Foundation (NSF), the MAA started a program called RUMC (Regional Undergraduate Mathematics Conferences), to seed new undergraduate mathematics conferences around the nation. PCUMC got started with funding from this program. Since then PCUMC "has lived up to our expectations and in fact has far exceeded them," wrote Colin Adams of Williams College in a letter supporting PCUMC for the Mathematics Programs That Make a Difference award. Adams is a co-principal investigator on the above-mentioned NSF grant and a founder of the Hudson River conference. The high of 650 PCUMC participants in 2012 was to his knowledge "the largest number of participants in any undergraduate mathematics conference that has yet occurred anywhere." In addition to the support from the MAA, PCUMC has received its own grants from the NSF, as well as funding from the National Security Agency and the Raytheon Company.

Held on the second Saturday of March, PCUMC rotates around institutions in the greater Los Angeles area and has been held at Occidental College, Loyola Marymount University, Pepperdine University, UC Riverside, Cal Poly Pomona, and California Lutheran University. Since its inception, the PCUMC has attracted over 2,400 participants and featured almost 400 student speakers representing 123 different institutions.

The conference puts special emphasis on bringing in women students and students who are members of underrepresented minorities. Close to 1,000 PCUMC participants have been women, 582 have been Latino/a or Hispanic, and 82 have been black or African American. PCUMC has developed strong ties with Hispanic-serving institutions and community colleges in southern California. One of the most important benefits of PCUMC is that it allows students to forge bonds with others who share their interest in mathematics-and such bonds can be especially important for students from underrepresented minorities. Often these students come from backgrounds where there is little support for pursuing higher degrees and where they are seen as unusual for their interest in mathematics. For such students to attend PCUMC and make contact with others like themselves causes a profound change in their outlook-they really view themselves as part of the mathematical community.

PCUMC not only has a direct positive impact on the students who attend. It also brings benefits to their institutions. Dana Clahane of Fullerton College first participated in PCUMC in 2010, when he brought a group of thirty students. "The response was nothing but long-lasting enthusiasm by the students who attended," he wrote in a letter supporting PCUMC's nomination for the award. "Attendance at our colloquia has ballooned since then ranging from ten to one hundred students and faculty each week, usually averaging approximately twenty-five.... PCUMC should be thanked for this miracle." Since 2010 Fullerton College has been a major participant in PCUMC. A majority of Fullerton students attending the conference have been women and members of underrepresented minorities. Most Fullerton participants are actually not mathematics majors. And this points to another positive feature of PCUMC: It helps cultivate in those who 
do not specialize in mathematics an appreciation of the beauty and usefulness of the subject.

PCUMC Directors: Alissa S. Crans, Loyola Marymount University, and Kendra Killpatrick, Pepperdine University

\section{About the Award}

CoProf created the Mathematics Programs That Make a Difference designation in 2005 as a way to bring recognition to outstanding programs that successfully address the issue of underrepresented groups in mathematics. Each year CoProf identifies one or two exemplary programs that:

(i) aim to bring more individuals from underrepresented minority backgrounds into some portion of the pipeline beginning at the undergraduate level and leading to an advanced degree and professional success in mathematics or retain them in the pipeline,

(ii) have achieved documentable success in doing so, and

(iii) are replicable models.

The CoProf subcommittee making the selection for this year's awards consisted of Lisa Traynor, David Savitt (chair), Robert Lazarsfeld, Deanna Haunsperger, and Javier Rojo.

Previously designated Mathematics Programs That Make a Difference are: the graduate program at the University of Iowa and the Summer Institute in Mathematics for Undergraduates/Research Experience for Undergraduates at Universidad de Puerto Rico, Humacao (2006); Enhancing Diversity in Graduate Education (EDGE) and the Mathematical Theoretical Biology Institute (2007); the Mathematics Summer Program in Research and Learning (Math SPIRAL) at the University of Maryland and the Summer Undergraduate Mathematical Science Research Institute at Miami University (Ohio) (2008); the Department of Statistics at North Carolina State University and the Department of Mathematics at the University of Mississippi (2009); the Department of Computational and Applied Mathematics at Rice University and the Summer Program in Quantitative Sciences, Harvard School of Public Health (2010); the Center for Women in Mathematics at Smith College and the Department of Mathematics at North Carolina State University (2011); the Mathematical Sciences Research Institute (2012); the Nebraska Conference for Undergraduate Women in Mathematics (2013); and the Carleton College Summer Mathematics Program and the Rice University Summer Institute of Statistics (the latter has moved to the University of Nevada at Reno) (2014).

-Allyn Jackson
AMERican Mathematical Society

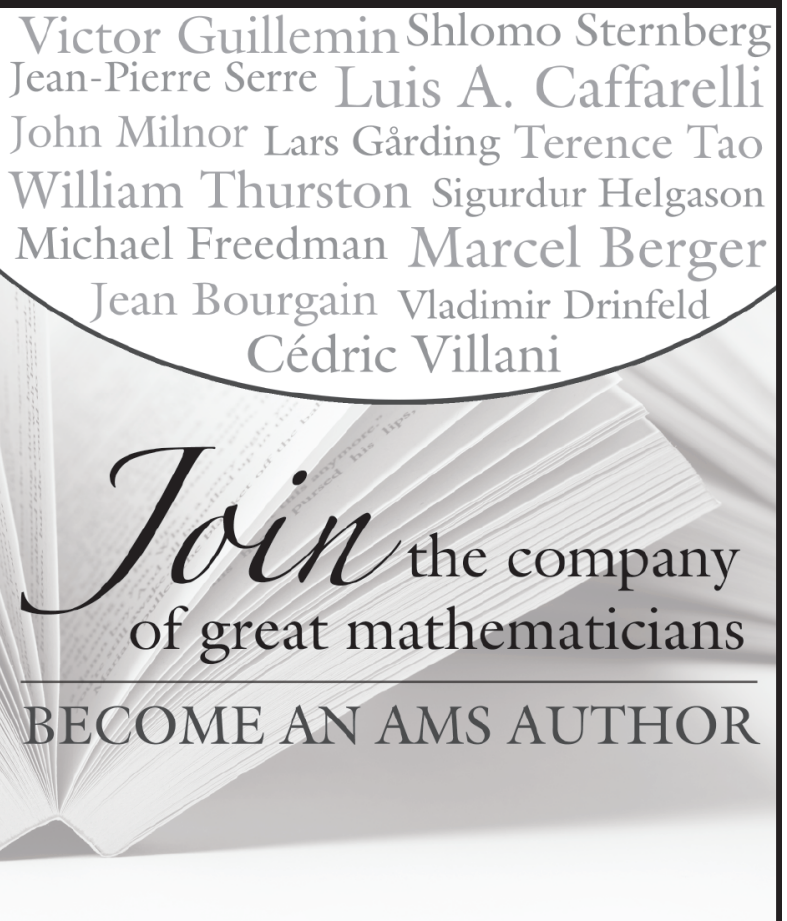

WHY PUBLISH WITH THE AMS?

We are mathematicians. The AMS is one of the world's leading publishers of mathematical literature. As a professional society of mathematicians, we publish books and journals for the advancement of science and mathematics. Consequently, our publications meet the highest professional standards for their content and production.

Expertise. Our editorial boards consist of experienced mathematicians. The AMS production staff is talented and experienced at producing high-quality books and journals. The author support group consists of experts in $\mathrm{TeX}$, graphics, and other aspects of the production of mathematical manuscripts.

Supporting mathematics. The AMS publication program is a part of our broader activities. The revenue it generates helps support our other professional activities. Thus, publishing with the AMS benefits the mathematical community.

Learn more at: www.ams.org/publications/ authors/becomeauthor

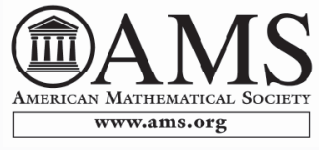

